to investigate the subject carefully and without prejudice, patiently developing what is good in the method, and gradually learning what is its proper place in the healing art.

Thus far its greatest value seems to have been in the treatment of early stages of phthisis, in fibroid phthisis, in chronic bronchitis, in emphysematous conditions of the lungs, and in the consolidations resulting from pleurisy, empyema, and unresolved pneumonia. Upon the Continent of Europe, the applications of compressed air have proved of inestimable value in the treatment of asthma, and it is not improbable that similar successes may soon be recorded here. lo meet these various indications, an ingenious mechanism attached to the Cabinet permits the operator at will either to rarefy the air within the ( $a b$ inet to a given degree, or to compress it to a given degree, or alternately to rarefy and compress it synchronously with the respiratory act.

Our only method of determining the merits of the treatment is by a careful study and a complete record of all cases. I trust to be able soon to report some original investigations upon the subject, studying it in its relations to and effect upon the bealthy organism as well as the diseased.

I94 West Brookline St., Boston, Mass.

\section{RUPTURE OF THE RECTUM, AND PROLAPSE OF THE SMALL INTESTINE. ${ }^{1}$}

BY S. B. LYON, M.D.,

GOVERNMENT HOSPITAL FOR THE INSANE, WASHINGTON, D, C.

I desire to present some notes on an unusual case of rupture of the rectum, and protrusion of a loop of the small intestine through the opening in the wall, and from the anus.

The patient was a woman of 5o, suffering with melancholia of a very active type, accompanied with delusions largely relating to the pelvic organs; she often fancied herself pregnant, or being delivered; and her conversation, which was almost continuous during waking hours, was on subjects forbidden in polite society. She had a remarkable talent for making remarks exceedingly embarrassing to both the physician and the nurses. One result of her delusive ideas of her pregnant condition, was a disposition at times to assist nature by introducing her hand into her vagina or rectum, and pulling or working at those organs, so that a tendency to prolapsus of both uterus and rectum existed, and these conditions had been reduced more than once. The disposition to interfere dangerously with herself made constant watchfulness and some restraint necessary.

A few days since, when visiting the ward, the nurse called my attention to a mass of viscera which had descended from the anus, and an examination showed at once that it was not a question of a prolapsus of the rectum, such as had previously occurred, but that the protruding mass was made up of a part of the smail intestine, and the appearance of omentum and the serous surface of the gut excluded a mere turning inside out. Only a rupture of the large intestine

IRead before the Medical Society of the District of Columbia, October 14,1885 . and the protrusion through the opening thus made of the small gut, could explain the condition. Th: rectum, with its internal and external sphincters, was in its nornial condition, not even slightly prolapsed by the weight and tension of the protruding mass.

Ordinary modes of reduction were attempted, but with no success; the weakened condition of the woman from shock, and long-continued exhausting excitement, precluded surgical interference. Within forty-eight hours death resulted without any sthenic symptoms. The following is Dr. Blackburn's report of the autopsy:

"Mrs. opening the abcomen a peritonitis of the folds of intestine in the polvic region was revealed. The peritoneal surfaces in that region were agglutinated by recent lymph, and a moderate quantity of reddish serum with floating lymph was contained in the cavity. On further examination a rupture of the anterior wall of the rectum within the recto-uterine pouch (Douglas's) was discovered. Through this opening a large loop of the ileum, with its mesentery, had passed. No escape of faces had occurred, as the intestine was adherent to the edges of the rupture. The portion of the intestine protruded began about six inches from the ileococal valve, and was about eighteen inches in length. The loop was discolored and covered with lymph. Some strangulation had occurred, shown by the thickening of the intestine and the discoloration of the part. Examination of the vagina and os uteri revealed an old laceration of the os, and erosion of the posterior lip of the cervix."

The only mention I have seen of a similar condition is under the head of hernia, as "Hedrocele," in Gross's "Surgery," where he quotes Dr. Uhde, of Brunswick; but no sack existing in the present case, it is doubtful if it is described under "Hedrocele."

\section{MEDICAL PROGRESS.}

\section{MATERIA MEDICA AND THERAPEUTICS.}

The Hippurates of IJime AND OF Jithitim.Dr. V. Poulet considers the hippurate of lime as the best preparation of calcareous salts for use in the system, and as especially useful in affections of the urinary passages; in affections of the liver; in diseases of the skin and mucous membrane, where they are dependent upon functional vices of the liver or upon lymphatism; in a great number of diseases of the alimentary canal, both of the stomach and intestine; in diabetes; and in chronic rheumatism, gout, etc.

In urinary affections, the subacute cystitis of the neck of the bladder yields satisfactorily to its use, with relief to the frequent desire to urinate, pain on urinating, etc. In certain cases where the urine manifests an abnormal alkaline reaction it becomes promptly acid again by the use of this drug. In every case, there is a return to the normal limpidity and a ressation of the presence of the mucous globules. It is quite as useful in urinary lithiasis from whatever cause, phosphaturia, uraturia, or oxaluria. If the theory of the 